\title{
DETERMINANTES SOCIOECONÓMICOS DE LA FRECUENCIA EN LAS RELACIONES SEXUALES EN POBLACIÓN UNIVERSITARIA
}

Jorge Montero-Mestre Universidad de Cartagena Colombia

Leider Ortiz-Caro Universidad de Cartagena Colombia

Robinson Castro-Avila Universidad de Cartagena Colombia 
Panorama Económico, Vol. 25 - No. 1 (Enero - Marzo de 2017), pp. 95-114

Jorge Montero-Mestre

Leider Ortiz-Caro

Robinson Castro-Avila

\title{
Determinantes socioeconómicos de la frecuencia en las relaciones sexuales en población universitaria
}

\section{Resumen}

El objetivo de este documento es evaluar algunos factores socioeconómicos, de índole social y en especial del grado de conocimiento y sensibilidad alrededor del tema de la sexualidad de la población joven universitaria, con la finalidad de brindar soporte a la necesidad o no de la puesta en marcha de campañas de prevención de factores de riesgo asociados a la actividad sexual. Los resultados indican que la edad del individuo, la edad de inicio de las relaciones sexuales, el consumo de alcohol y la puesta en práctica de técnicas de planificación, inciden en la frecuencia sexual de los jóvenes universitarios. Se concluye que la población joven pondera en mayor medida los riesgos a tener un embarazo no deseado que a las enfermedades de transmisión sexual.

Palabras clave: Sexualidad, Adolescentes, Fecundidad, Universitarios.

Clasificación JEL: C35, C51, I19

\section{Socioeconomic determinants of sexual relations frequency in university population}

\begin{abstract}
This research analyze socioeconomic factors, and sexuality awareness among undergraduate students in order to evaluate the implementation (or not) of prevention public campaigns associated to sexuality. Results indicate that age of individuals, their starting age of sexual activity, alcohol consumption and family planning affect sexual relations frequency of undergraduate students. In addition, youngsters consider most the risks of unwanted pregnancy than sexually trasnmitted diseases.
\end{abstract}

Keywords: Sexuality, fertility, undergraduate students

JEL Classification: C35, C51, I19

\section{Déterminants socioéconomiques de la fréquence des relations sexuelles dans la population universitaire}

\section{Résumé}

Cette recherche analyse les facteurs socioéconomiques et la sensibilisation à la sexualité chez les étudiants de premier cycle afin d'évaluer la mise en œuvre (ou non) de campagnes publiques de prévention associées à la sexualité. Les résultats indiquent que lâge des individus, leur âge de début d'activité sexuelle, la consommation d'alcool et la planification familiale affectent la fréquence des relations sexuelles des étudiants de premier cycle. En outre, les jeunes considèrent plus les risques de grossesse non désirée que les maladies sexuellement transmissibles.

Mots-clés: Sexualité, fertilité, étudiants de premier cycle.

Nomenclature JEL: C35, C51, I19 


\title{
Determinantes socioeconómicos de la frecuencia en las relaciones sexuales en población universitaria
}

\author{
Jorge Montero-Mestre* \\ Universidad de Cartagena, Colombia \\ Leider Ortiz-Caro \\ Universidad de Cartagena, Colombia \\ Robinson Castro-Avila \\ Universidad de Cartagena, Colombia
}

\section{INTRODUCCIÓN}

El comportamiento sexual en la población joven, en especial en la población universitaria puede estar determinada por factores socioeconómicos, de índole social y en especial por el grado de conocimiento y sensibilidad alrededor del tema de la sexualidad. Cuando se tiene en cuenta la población joven que ingresa a las universidades, en su mayoría, se observan conductas que están enfocadas en buscar aceptación en un determinado grupo social en donde las prácticas, en especial las prácticas sexuales, pueden ser objeto de observación para la vinculación a determinados círculos sociales. Por lo que se demuestra que los pares influyen junto con la concepción dominante que se encuentra en la sociedad. (Fishbein \& Ajzen, 1975; Leland \& Barth, 1993; Bearman \& Brückner, 1999).

Experiencias de investigación en la Universidad de Manizales que estudian las prácticas y comportamientos sexuales de la población estudiantil (Constanza et al., 2010), en el cual se identifican los determinantes con mayor significancia estadística, los cuales pueden ser la edad, los ingresos, la aversión a ETS, el estado civil junto al de los padres, como medida aproximada de la composición del hogar, los gastos para mantener las prácticas sexuales y el consumo de alcohol.

Asimismo, al analizar este factor de práctica social, se encuentran muchos mitos y riesgos infundados para las personas involucradas, mucho más en los jóvenes. Hoy la juventud se

\footnotetext{
* Autor para correspondencia

Correos electrónicos: jmonterom@unicartagena.edu.co*, lortizc@unicartagena.edu.co, rcastroavila@yahoo.es Artículo dado como resultado de trabajos de campo, en el marco de actividades de aula del curso de Econometría (2016), se agradecen los comentarios de Álvaro Andrés Escobar y de Bernardo Romero.
} 
encuentra frente a la llamada sociedad del riesgo, caracterizada por una condición de incertidumbre, violencia y amenazas; por lo cual es necesario entender y explorar las experiencias de estos. Un mito existente entre los jóvenes vinculado a las relaciones sexuales es que "entre los hombres quiénes tienen mayor nivel de ingresos, recursos o dotación, pueden tener mayor frecuencias de relaciones sexuales", dado que este tipo de hombres tienden a ser más portentoso por medio del dinero, es decir se tienen en cuenta las posesiones que los hombres tienen, como los vehículos, apartamentos, empresas, etc. (Constanza et al., 2010).

Otro mito sobre las relaciones sexuales que radica en la sociedad es que "los jóvenes que poseen mayores relaciones sexuales, están expuestos a las enfermedades de transmisión sexual" una afirmación que puede ser cierta en un país donde el 37,1\% de las mujeres y el $33,7 \%$ de los hombres entre 13 a 49 años de edad, en el año 2015 manifestó no tener conocimiento comprensivo sobre el VIH/SIDA (Ministerio de Salud y Protección Social, 2016).

Rodríguez et al. (2006) (citado por Constanza et al., 2010) plantea que, entre las condiciones y situaciones que aumentan la vulnerabilidad en la población universitaria están: el inicio de relaciones sexuales a edades más tempranas, parejas sexuales ocasionales, resistencia al uso del preservativo y la falta de información. Por lo anterior, se espera que los universitarios que no recibieron algún tipo de educación sexual se consideren vulnerables a las enfermedades de trasmisión sexual y por ende exista una relación negativa con la frecuencia de las relaciones.

La pertinencia de esta investigación radica en sentido general, en el entendimiento de cómo es la dinámica o comportamiento de las prácticas sexuales de una población universitaria, con el propósito, principalmente, de brindar soporte a la necesidad o no de la puesta en marcha de campañas de prevención de factores de riesgo asociados a la actividad sexual, como lo son las ETS y el aborto. De esta forma, el objetivo de la presente investigación es conocer los diferentes factores que a la luz de la literatura encontrada inciden en la frecuencia de las relaciones sexuales. Para cumplimiento de esto, se emplean técnicas econométricas a partir de datos de recolectados por encuestas aplicadas a la población universitaria de la facultad de ciencias económicas de la Universidad de Cartagena.

El documento se divide en seis secciones, siendo la primera esta introducción, seguida de una revisión de la literatura referente a la temática tratada en esta investigación. Posteriormente se sustentan la composición de los datos y la metodología aplicada en el presente trabajo, y por último se discuten los resultados obtenidos y se ofrecen algunas conclusiones.

\section{EL COMPORTAMIENTO SEXUAL EN LOS JÓVENES}

Sobre las prácticas y comportamientos sexuales de los jóvenes se han desarrollado diversas investigaciones direccionadas a grupos de adolescentes en educación media y superior; permitiendo ver la influencia de diferentes variables como género, familia, religión y formación educativa en el comportamiento y la práctica sexual responsable.

Según Piña \& Rivera (2009) "cuando en sociedades como la nuestra se refuerzan estereotipos acerca de que la mejor "conquista" es la que se realiza cuando alguien se involucra con personas atractivas del 
género opuesto, la gratificación inherente a dicha práctica, sumada a la eventual gratificación obtenida en la relación sexual como tal, no hacen sino fortalecer la tendencia a reproducir dicho comportamiento cada vez que una persona lo considere oportuno y pertinente".

Dentro de la etapa de la adolescencia es donde se centra la mayor parte del análisis de esta temática, para esto Barrera, Sarmiento, \& Trujillo (2004) dan a entender la relevancia de este tema. Por tal razón, actualmente se reconoce que durante la adolescencia las personas tienden a practicar diferentes formas de actividad sexual. Diamond, Savin-Williams y Dubé (1999) definen la actividad sexual como un continuo de comportamientos motivados por el deseo sexual y orientado hacia el placer y la gratificación independientemente de que culmine o no en el orgasmo.

Entender este comportamiento, significa entender el entorno en el cual se ha desenvuelto la persona misma, teniendo en cuenta así factores sociales, familiares, culturales, económicos, emocionales, etc. Es por tanto que Barrera, Sarmiento \& Trujillo (2004) plantean que las actitudes parentales conservadoras respecto al comportamiento sexual adolescente se relacionan significativamente con un menor número de relaciones sexuales genitales y de compañeros sexuales.

Adicional a esto, otros investigadores han encontrado que la actividad sexual de los adolescentes está influenciada por el comportamiento del mejor amigo o amiga, o de la persona con la cual se tiene una relación estable o romántica (Miller, McCoy \& Olson, 1986; Small \& Luster, 1994; Gaston, Jensen, \& Weed, 1995), influyendo de esta manera en la pronta edad para el inicio de las relaciones sexuales, como sustentan Perkins, Luster, Villarruel, \& Small (1998) de que la edad de la primera relación sexual se puede explicar, en parte, por la influencia del grupo.

De acuerdo con Kinsman et al (1998) la motivación principal de los adolescentes para iniciar su actividad sexual, no es que sientan que es divertida, sino que no quieren quedarse atrás con respecto al grupo de adolescentes de su misma edad. En Colombia según Holguin et al (2012) la edad media de inicio de relaciones sexuales es de aproximadamente 15 años, que se distingue por ser la etapa de crecimiento y asociación social activa de la persona.

Para Singh \& Wulf (1990) se aprecian como significativas las condiciones socioeconómicas que se han identificado como el nivel educativo, el nivel de urbanización, el nivel de ingreso, factores familiares y factores culturales, de tal forma que en el estudio de Baumer \& South (2001) se encontró que en estratos bajos, los adolescentes hombres promueven el inicio temprano de relaciones y una alta frecuencia de actividad sexual, para adquirir prestigio y estima. Así mismo los embarazos no planeados se pueden asumir como un mecanismo para ganar estatus. En el caso de los hombres porque aumenta su sentido de masculinidad (Marsiglio, 1993) $\mathrm{y}$ en el de las mujeres porque adquieren respeto social como madres (Stern, 2002)

En esta misma dirección, Flórez (2005) sustenta que las adolescentes del estrato bajo comienzan la maternidad a menor edad y se incorporan a ella con mayor intensidad que las del estrato alto. Así, el nivel y patrón de la fecundidad en la adolescencia son mucho mayores en el estrato bajo que en el estrato medio alto. 
Es de destacar que la planificación y la educación sexual en los últimos años ha aumentado en importancia, esto se demuestra en la lucha que ha tenido el gobierno por impulsar estas medidas como control de prevención, estas medidas del gobierno colombiano tienen su origen, en 1998, en donde se establecen los lineamientos para una política de salud sexual y reproductiva, incorporando el derecho a una educación sexual y reproductiva desde la infancia y el derecho a servicios de salud sexual y reproductiva integral (MinSalud, 1998), lo que resulta muy importante al considerar lo que plantea Flórez (2005), de que las políticas y programas gubernamentales respecto al sector de la salud y otros relacionados que se aplican a través de campañas de planificación familiar y promoción del acceso a la educación formal y a la educación sexual son importantes a la hora de prevenir o controlar los efectos negativos de un descontrol de esto. Además demuestra que algunos otros factores como el contexto social y del hogar en el que vive el adolescente, y características individuales, son muy relevantes a la hora de intentar entender este comportamiento en la población joven.

Es por esto que se esperaría que el efecto de exposición al riesgo de embarazo sea menos importante entre mayor sea la aceptación y el uso de métodos de planificación familiar. Igualmente entre mayor sea la exposición al riesgo de embarazo y menor sea el acceso a métodos de planificación familiar, mayor la importancia al aborto.

Para Flórez et al. (2004) aunque la planificación familiar juega un papel importante, su uso empieza después de que han iniciado relaciones sexuales. Esto se basa en la percepción de invulnerabilidad que tienen los adolescentes, en la creencia infundada de efectos secundarios, y en la creencia que no se necesitan métodos de control en la primera relación, o básicamente en el desconocimiento o inmadurez respecto a las relaciones sexuales. A pesar de esto, los esfuerzos parecen ser nulos, puesto que para el estudio efectuado por Flórez et al. (2004) se evidencia el efecto limitado que sobre el comportamiento de los adolescentes ha tenido la educación sexual que se imparte en los colegios desde 1993.

Aunque el conocimiento de planificación familiar parece no ser bajo entre las adolescentes el nivel de uso de métodos modernos, especialmente entre las adolescentes sexualmente activas pero no en unión permanente, es muy bajo en la mayoría de los países Latinoamericanos con excepción de Colombia en donde un 10\% de las mujeres de 15-19 años usaron algún método contraceptivo, siendo para 2010 que siete de cada 10 mujeres entre edades de 15 a 24 años que mantuvieron relaciones sexuales durante el último año no habían utilizado preservativo en su última relación (UNICEF, 2015).

Respecto al nivel de uso, se observa que las mayores tasas de no uso de preservativos se encuentran en la población Rural, entre las mujeres de entre 20 a 24 años y quienes se encuentran en los percentiles más bajos en los indicadores de bienestar. Esos bajos valores de uso entre las adolescentes, $\mathrm{y}$ especialmente, $\mathrm{y}$ posiblemente entre las solteras, indican la posible existencia de barreras para el uso de métodos de planificación entre las jóvenes. Esas barreras podrían incluir la falta de información en el uso adecuado de los métodos, y/o dificultades en el acceso a los métodos (ya sea por barreras culturales o costos) (Blanc \& Way, 1998) 
Entre los factores de riesgo más relevantes de las ETS, descritos por la literatura, se encuentran el consumo de sustancias alucinógenas, tabaco y bebidas alcohólicas (Tamayo et al., 2008) que propician el sexo sin protección y múltiples parejas sexuales; además la falta de información y la educación en salud. La encuesta nacional de demografía y salud (ENDS) realizada por Profamilia en el año 2000, reportó que el nivel educativo y el lugar de residencia pueden incidir en las decisiones sexuales; Se encontró que los creyentes inician más tardíamente sus relaciones sexuales, aunque más de la mitad presentan prácticas como la masturbación en un 55,5\%. (Constanza et al, 2010)

Dentro de esta investigación (Constanza et al, 2010), se encuentran factores en los cuales se considera que una cuarta parte de las ETS reportadas en el mundo, suceden en adolescentes, entre las causas figura la carencia de información que conlleva a asumir riesgos en los contactos sexuales entre los resultados de esta investigación, se obtuvieron que de entre los factores más frecuentes de las mujeres se encuentra la frecuencia diario y entre tres veces a la semana en las relaciones sexuales $(13,6 \%)$ y además, otro hallazgo importante fue la alta prevalencia de factores de riesgo relacionados con las prácticas sexuales, sobresale que el $56 \%$ de los escolares en estudio tuvieron su primera relación sexual antes de los quince años, siendo mayor en los hombre comparado con las mujeres. En la encuesta Nacional de Salud (ENS) del 2010, en Colombia el 13\% de las mujeres iniciaron relaciones sexuales antes de los 15 años, a diferencia de este estudio que fue del 40,2\%; por tal motivo, los programas de educación sexual deberían iniciar en edades más tempranas con el objetivo de retardar la edad de inicio en las relaciones sexuales o en caso tal, estas sean asumidas con mejor nivel de educación e información.
Para los casos colombianos, y según las encuestas de Demografía y Salud, indican que la incidencia de la fecundidad en adolescentes ha venido aumentando, junto a una tasa creciente de embarazos $\mathrm{y}$ nacimientos prematrimoniales $\mathrm{y}$ un aumento significativo del inicio temprano de la actividad sexual. (Flórez, 2005).

\section{MARCO TEÓRICO}

Lasteoríasqueenmarcan el rol sexual como un aspecto adicional al comportamiento de la persona, ya sea desde su dimensión social o psicológica, son las que de acuerdo al análisis ofrecido aquí sirven de ayuda para entender la dinámica social de los jóvenes entorno a esta temática, es por tanto que guiándonos de Consol Cabral (1983) analizamos aquellas teorías que refuerzan la explicación de estos comportamientos.

\subsection{Teoría del refuerzo directo}

Cuyo máximo exponente es Mischel W. el cual analiza la relevancia de las respuestas parentales ante la conducta del rol sexual de niños y niñas, y sostiene que los padres, mediante refuerzos, determinan, desde los primeros meses de vida, las respuestas y el comportamiento de sus hijos, entendiéndose esta como la educación respecto a los principios y valores que se aprenden en el hogar, y que además está asociada a la estructura familiar.

\subsection{La Teoría de la Acción Razonada(TAR)}

Fishbein \& Ajzen (1975) nos ofrece los elementos conceptuales para contrastar que las actitudes personales y la norma social percibida pueden operar como factores explicativos de la actividad sexual de los adolescentes. Los antecedentes empíricos sobre la actividad sexual de los 
adolescentes señalan que las actitudes personales y de las personas cercanas influyen sobre la disposición a tener relaciones sexuales (Leland \& Barth, 1993) (Bearman \& Brückner, 1999).

Asimismo, esta teoría plantea que el antecedente inmediato de cualquier comportamiento (la actividad sexual) es la intención de ejecutarlo (la disposición para tener relaciones sexuales penetrativas durante la adolescencia). Concretamente, en el contexto de este estudio se plantea que esta intención conductual está determinada, a su vez, por a) la tendencia favorable o desfavorable del adolescente hacia el comportamiento (actitud personal hacia la actividad sexual en la adolescencia); b) la percepción del joven sobre las actitudes de otros significativos frente al hecho de realizar el comportamiento (percepción del adolescente de qué tan favorable es la opinión de los padres y de los amigos hacia el hecho de tener relaciones sexuales en la adolescencia) y por último c) la percepción del número de miembros del grupo de pares que practican la conducta, en otras palabras, percepción de prevalencia o norma de pares. La percepción de las actitudes de los otros significativos y la norma de pares constituyen lo que se ha denominado norma social percibida.

\subsection{La Teoría del desarrollo cognitivo del rol sexual}

Está basada en el supuesto de que el patrón básico de las actitudes sexuales no se puede encontrar ni en los instintos biológicos ni en las normas culturales arbitrarias, sino en factores cognitivos. Es una teoría basada en Piaget que pone de relieve, sobre todo, los aspectos de curiosidad, exploración y competencias del joven y cuyo máximo exponente es Kohlberg (1966).

\section{DATOS Y ESTADÍSTICAS DESCRIPTIVAS}

\subsection{Datos}

La recolección de los datos se realizó por medio de una encuesta virtual, que a partir de información brindada por los departamentos que componen la facultad de ciencias económicas, se logró obtener los correos electrónicos de los estudiantes a los cuales se les hizo llegar la encuesta, con la finalidad de que la encuesta fuera llenada con total anonimidad de lo reportado por el encuestado, con lo que se garantizaría que los resultados aquí brindados son lo más acercados a la realidad de la población universitaria, en específico la población de la facultad de ciencias económicas.

Siguiendo a Martínez-Bencardino (2012) se calculó una muestra óptima de 300 estudiantes, de una población de 1600 estudiantes, respecto a la participación de la población de cada departamento en el total de la facultad, así se tuvo en cuenta la ponderación en el cálculo de la muestra por cada programa académico.

Los tipos de variables empleadas en esta investigación son inicialmente seis de tipo cuantitativo discretas y ocho variables cualitativas, que dentro del análisis pasan a ser representadas como variables dicotómicas, que toman el valor de uno en el aspectodereferencia, y el valor deceroen otro caso. Estas variables son: la frecuencia en las relaciones sexuales en el último semestre a la hora de ser encuestado (2016), la edad de la persona encuestada, la edad en la que tuvo su primer encuentro sexual, el nivel de ingresos mensuales, los gastos asociados en los que se hacen necesario incurrir a la hora de realizar la práctica sexual, el género, frecuencia con material sexo-excitante, estado civil de la persona encuestada y el estado civil de los padres, 
como proxy de la estructura familiar, la manifestación de ser creyente o no, el consumo regular de bebidas embriagantes, la percepción de vulnerabilidad ante las enfermedades de transmisión sexual (ETS) y por último la puesta en práctica de técnicas de planificación como medida de prevención de embarazos no deseados, esto si la persona manifiesta llevar una vida sexual activa.

\subsection{Estadísticas descriptivas}

Las estadísticas descriptivas de nuestros datos se reportan en la Tabla 1

En donde para una muestra de 300 observaciones en promedio se reporta una edad de 21 años, entre 17 y 27 años que es el margen de edad evaluado. Dentro de este rango se tiene que en el último semestre los estudiantes en promedio tuvieron 5 relaciones sexuales, entre $0 \mathrm{y}$ 18 veces, entre los cuales la edad de inicio gira entorno a los 16 años de edad, aunque hay quienes lo comenzaron desde los 13 hasta los 27 años (ONU, 2006).

La muestra obtenida, de acuerdo a las variables analizadas, se componen por $58,33 \%$ de hombres y $41,67 \%$ mujeres; el $60 \%$ se encuentran en una relación estable y el $40 \%$ no; también de esta muestra el $58 \%$ de los estudiantes provienen de un hogar con padres casados y el $42 \%$ de padres no casados; adicionalmente, el $75 \%$ manifiesta creer en un Dios, y aprueba el estar vinculado a un religión teísta (Gráfica 1, ver anexo).

Por otra parte, respecto a la educación sexual recibida en el hogar, $70 \%$ de los encuestados manifiesta no haberla recibido contra un 30\% que sí, de ahí la razón de observar que el $76 \%$ manifestó no sentirse vulnerable de adquirir una enfermedad de transmisión sexual (ETS), aunque se observa que aquellos que manifiestan ya tener una actividad sexual regular, el $61 \%$ planifica y el $39 \%$ no lo hacen, lo que podría dar una imagen a que la juventud actual considera más importante, el prevenirse de embarazos no deseados que de las ETS.

La media en las relaciones sexuales por género, resulta ser mayor en los hombres que en las mujeres. La Gráfica 3 (ver anexo) muestra que por sexo, el promedio de relaciones en los hombres en el último semestre fue de 6 contra 4 relaciones por parte de las mujeres, sin embargo se observa que en los hombres disminuye el promedio de relaciones a medida que aumenta la edad, contrario a lo que sucede en las mujeres, que va aumentando. En cuanto a la edad de inicio, se tiene un comportamiento más parejo entre hombres y mujeres, en donde ambas son mayores a medida que la edad de inicio es mayor.

Tabla 1. Estadísticas descriptivas de las variables cuantitativas del modelo.

\begin{tabular}{|l|c|c|c|c|c|}
\hline \multicolumn{1}{|c|}{ Variable } & Obs & Media & Desv. Est. & Min & Max \\
\hline $\begin{array}{l}\text { Frecuencia en las relaciones } \\
\text { sexuales }\end{array}$ & 300 & 5.05 & 5.01 & 0 & 18 \\
\hline Edad & 300 & 20.96 & 2.09 & 17 & 29 \\
\hline Nivel de Ingresos & 300 & $\$ 362,500$ & $\$ 412,049$ & 0 & $\$ 1,250,000$ \\
\hline Gastos en Actividad Sexual & 300 & $\$ 36,250$ & $\$ 45,358$ & 0 & $\$ 175,000$ \\
\hline Edad de inicio & 300 & 15.69 & 6.62 & 13 & 27 \\
\hline $\begin{array}{l}\text { Frecuencia con Material } \\
\text { Sexo Excitante }\end{array}$ & 300 & 3.90 & 4.36 & 0 & 18 \\
\hline
\end{tabular}




\section{METODOLOGÍA}

\subsection{Estimación de los determinantes de la frecuencia sexual en los universitarios}

A partir de un modelo de análisis de covarianza, se evalúan las hipótesis que la literatura aborda respecto a la conducta sexual de los jóvenes universitarios. Se estima el siguiente modelo con los datos recolectados:

\subsection{Probabilidad respecto al nivel de frecuencia sexual}

Para ampliar el análisis se realiza, por medio de un modelo logístico ordenado, una evaluación del cambio en la probabilidad de tener una baja, media o alta frecuencia sexual entre los jóvenes universitarios, a partir de un grupo de variables, que nos permiten contrastar una vez más su efecto sobre la variable de

$$
\begin{aligned}
& \text { Frec }_{i}=\beta_{0}+\beta_{1} E d_{i}-\beta_{2} E d_{i}^{2}+\beta_{3} G_{i}+D_{1} E d I_{i}+D_{2} E C_{i}+D_{3} E C P_{i}+D_{4} C_{i}+D_{5} P l_{i}+\mu_{i} \\
& \text { Donde: } \\
& F_{E r e c}=\text { Frecuencia sexual } \\
& E d_{i}=\text { Edad } \\
& E d_{i}^{2}=\text { Edad al cuadrado } \\
& G_{i}=\text { Gasto incurridos durante la práctica sexual. } \\
& E d I_{i}=\text { Edad de inicio de relaciones sexuales. } \\
& E C_{i}=\text { Estado Civil } \\
& E C P_{i}=\text { Estado Civil de los Padres. } \\
& C_{i}=\text { Creencia } \\
& P l_{i}=\text { Planificación } \\
& \mu_{i}=\text { Error estocástico que se asume con media cero y varianza constante. }
\end{aligned}
$$

interés, ya no esta vez sobre la varianza de la variable dependiente, sino respecto a la probabilidad.

Para lo anterior, se reestructuró la variable dependiente (Frecuencia en las relaciones sexuales en el último semestre), convirtiéndola en una variable categórica ordenada (Baja, Media y Alta frecuencia).

\section{RESULTADOS}

Los resultados obtenidos son los presentados en la Tabla 2 (ver anexo), en donde se observan los coeficientes de acuerdo a su significancia y según el modelo de mínimos cuadrados (MCO) y mínimos cuadrados generalizados (MCG) ${ }^{1}$. De acuerdo a lo anterior, comprobamos que la edad de las personas encuestadas, además de la edad de comienzo de las relaciones sexuales, tienen un efecto sobre la frecuencia en las relaciones sexuales. Adicionalmente, como lo postula la literatura consultada romántica (Miller, McCoy \& Olson, 1986; Small \& Luster, 1994; Gaston, Jensen, \& Weed, 1995), se puede argüir que aquellas personas que reportan una mayor edad, son aquellas que reportaron una edad de inicio de relaciones más tardía, lo que nos permite

1 Este último se presenta con el objetivo de controlar la heteroscedasticidad en los errores, además se debe tener presente de que los resultados presentados son robustos. 
extraer la conclusión, que las relaciones más modernas, están asociadas a un contacto con la vida sexual a más temprana edad, que las generaciones un poco más anteriores, teniendo en cuenta que las poblaciones más actuales suelen ser mayormente sociables, como lo reporta la Gráfica 2 (ver anexo).

Soportamos estos resultados, con los reportados en la Gráfica 5 (ver anexo), en donde se observa que la máxima probabilidad se encuentra en el rango de edad de inicio de 16 a 20 años, de encontrarse en una frecuencia baja de relaciones, disminuyendo al aumentar la edad, mientras que aumenta la probabilidad de encontrarse en una media y alta frecuencia, independientemente de encontrarse en una relación estable o no, y además independiente de provenir de una familia en donde los padres se encuentran casados o no. Lo que nos permite concluir que aquellas personas que comienzan a mayor edad sus relaciones sexuales, lo hacen para mantenerse en una actividad constante.

Al evaluar aquellos variables que Constanza et al., (2010) en su trabajo expone para la Universidad de Manizales, encontramos que el consumo de alcohol está asociada con una mayor frecuencia en las relaciones sexuales, que como exponen Tamayo et al., (2008) el mayor consumo de alcohol propicia la actividad sexual tanto sin protección como con múltiples parejas, dentro de esta misma literatura, vemos que la planificación juega un papel fundamental en la decisión de tener una vida sexual activa o no, como medida de prevención de embarazos no deseados, por lo que encontramos que si la persona manifiesta planificar, esto está asociado con un menor nivel de relaciones sexuales en comparación con aquellas personas que no lo realizan, esto se explica por el carácter de mayor consciencia que tienen las personas que manifiestan planificar, independientemente del número de relaciones que tengan en promedio, son más conscientes respecto a la probabilidad de tener un embarazo no deseado que aquellos que no planifican, es decir, la decisión de planificar o no, en si no juega un papel relevante en las decisiones más que el de tener una mayor consciencia y por ende un mayor control en su conducta y/o actividad sexual.

\section{CONCLUSIONES}

En el intento de cumplir con el objetivo de contrastar algunas teorías y aportes empíricos respecto a la actividad sexual en la población joven, en específico de la universidad de Cartagena, en su Faculta de ciencias económicas, podemos concluir que de acuerdo con Kinsman et al (1998) la edad de la persona está asociada con un mayor grado de sociabilidad, lo que según con la teoría de la acción razonada, declara a esta población más vulnerable respecto al grado de contacto con la actividad sexual, por factores de grupo o de pares, lo que se refleja en su relación directa con la edad de inicio de relaciones sexuales (Miller, McCoy \& Olson, 1986; Small \& Luster, 1994; Gaston, Jensen, \& Weed, 1995; Perkins, Luster, Villarruel, \& Small, 1998).

Asi mismo, se encontró que el consumo de alcohol y la planificación contribuyen a explicar el grado de actividad sexual entre este grupo de jovenes, extrayendo como conclusión, al tener en cuenta la vulnerabilidad a las ETS, que los adolescentes responden mayormente a los riesgos asociados a los embarazos no deseados que a las enfermedades de trasnsisión sexual, lo que se puede explicar en parte, por la baja tasa de reporte de 
educación sexual recibida y porque un embarazo significaría poner en peligro la continuidad de los estudios universitarios.

Queda para futuras investigaciones el poder tener en consideración, con variables más apropiadas, el componente de asociación de los jóvenes respecto a la percepción de actividad sexual, debido que de acuerdo al marco literario y teórico de este trabajo, dicho factor es de gran importancia a la hora de tratar de entender el comportamiento sexual de los jóvenes.

\section{REFERENCIAS BIBLIOGRÁFICAS}

Barrera, F., Sarmiento, E., \& Trujillo, E. V. (2004). Relación de las actitudes personales y de la norma social con la actividad sexual de los adolescentes. Revista de estudios sociales, 56-66.

Baumer, E., \& South, S. (2001). Community Effects on Youth Sexual Activity. Journal of Marriage and the Family, 540-554.

Baumer, E., \& South, S. (2001). Community Effects On Youth Sexual Activity. . Journal of Marriage and the Family , 540-554.

Bearman, P., \& Brückner, H. (1999). Power in Numbers: Peer Effects on Adolescent Girls' Sexual Debut and Pregnancy. National Campaign to Prevent Teen Pregnancy.

Consol Cabral, M. (1983). Nuevas aportaciones al estudio sobre el rol sexual: La teoría del esquema cognitivo del sexo. Quaderns de Psicologia, 41-53.

Constanza Cañón, S., Castaño Castrillón, J. J., Alejandra Díaz, S., Hernández, J. A., Hoyos Martínez, Y., López Marín, F.,...Ortega, A. M. (2010). Prácticas y comportamientos sexuales de estudiantes de la Universidad de Manizales. psicología desde el caribe.

Diamond, L., Savin-Williams, R., \& Dubé, E. (1999). Sex, Dating, Passionate Friendships and Romance: Intimate Peer Relations among Lesbian, Gay and Bisexual Adolescents. (C. U. Press., Ed.) The Development of Romantic Relationships in Adolescence.

East, P. (1996). The Younger Sisters of Childbearing Adolescents: Their Attitudes, Expectations, and Behaviors. Child Developmen, 267-282.

Fishbein, M., \& Ajzen, I. (1975). Belief, Attitude, intention and behavior: An introduction to Theory and Research. Addison -Wesley.

Flórez, C. E. (2005). Factores socio económicos y contextuales que determinan la actividad reproductiva de las adolescentes en Colombia. Panam Salud Pública.

Flórez, C. E., Vargas, E., Henao, J., Gonzalez, C., Soto, V., \& Kassem, D. (2004). Fecundidad Adolescente en Colombia: Incidencia, tendencia y determinantes. Un enfoque de historia de vida. CEDE.

Florez, C., \& Nuñez, J. (2000). Teenage Childbearing in Latin American Countries . IADB, Research Network Working Paper, R-434. 
Gaston, J., Jensen, L., \& Weed, S. (1995). A Closer Look At Adolescent Sexual Activity. Journal of Youth and Adolescent , 465-479.

Holguin, Y. P., Mendoza, L. A., Esquivel, C. M., Sánchez, R., Daraviña, A. F., \& Acuña, M. (2012). Factores asociados al inicio de la actividad sexual en adolescentes de Tuluá, Colombia. Revista Chilena de Obstetricia y Ginecologia, 209-219.

Kinsman, S., Romer, D., Furstenberg, F., \& Schwarz, D. (1998). Early Sexual Initiation: The Role of Peer Norms. Pdiatrics, 1185-1192.

Kinsman, S., Romer, D., Furstenberg, F., \& Schwarz, D. (1998). Early Sexual Initiation: The Role of Peer Norms. Pediatrics, 1185-1192.

Kohlberg, L. (1966). A cognitive developmental analysis of children's sex-role concepts and attitudes. Stanford, Stanford University Press, 82-173.

Leland, N., \& Barth, R. (1993). Characteristics of Adolescents who have Attempted to avoid HIV and who have Communicated with Parents about Sex. Journal of Adolescent Research, 58-76.

Magnani, R., Seiber, E., Zieliski Gutierrez, E., \& Vereau, D. (2001). Correlates of Sexual Activity and Condom use among Secondary - School Students in Urban Peru. Studies in Family Planning , 53-66.

Martínez-Bencardino, C. (2012). Estadística y Muestreo . ECOE Ediciones

Marsiglio, W. (1993). Adolescent Males Orientation Toward Paternity and Contraception. Family Planning Perspectives, 22-31.

Miller, B., McCoy, J., \& Olson, T. (1986). Dating Age And Stage as Correlates of Adolecent Sexual Attitudes And Behavior. Journal of Early Adolescent Research, 361-371.

Ministerio de Salud y Protección Social. (12 de 12 de 2016). Ministerio de Salud y Protección Social. Obtenido de Ministerio de Salud y Protección Social:https://www.minsalud.gov.co/Paginas/ Ministerio-de-Salud-y-Profamilia-entregan-resultados-de-la-ENDS-2015.aspx

MinSalud. (1998). Lineamientos de políticas de salud sexual y reproductiva. Documento Técnico, No. 1.

Perkins, D., Luster, T., Villarruel, F., \& Small, S. (1998). An Ecological, Risk Factor Examination Of Adolecents' Sexual Activity in the Three Ethnic Gropus. Journal of Marriage and The Family., 660-673.

Piña López, J. A., \& Rivera Icedo, B. M. (2009). Predictores del comportamiento sexual con múltiples parejas en estudiantes de educación superior: Un análisis por género. Acta Colombiana De Psicología, 53-65.

Rodríguez, F., Primo, S., Martínez-Calvo, E., \& Sánchez, M. (2006). Sida/ ITS y sexualidad en ingresantes universitarios. Ciencia Docencia Tecnología , 135-150.

Singh, \& Wulf. (1990). Adolescentes de hoy, Padres del mañana: Un perfil de las Américas. Nwe Cork: Alan Guttmatcher Institute. 
Small, S., \& Luster, T. (1994). Adolescent Sexual Activity: An Ecological, Risk Factor Approach. Journal of Marriage and the Family, 181-192.

Stern, C. (2002). Poverty, Social Vulnerability and Adolescent pregnancy in México: A Qualitative Analysis. (B. C. University, Ed.) Investigación presentada en el CICRED, Seminar on Reproductive Health, Unmet Needs, and Poverty: Issues of Access and Quality of Services.

Tamayo Acevedo, L. S., Lopez, M. I., Villegas, A., Agudelo, C., Arrubla, M., \& Muñoz Tamayo, J. (2008). Determinantes de Salus Sexual e ITS en Adolescentes Rurales, Escolarizados. Salud Pública de Medelin .

UNICEF. (2015). UNA APROXIMACIÓN A LA SITUACIÓN DE ADOLESCENTES Y JÓVENES EN AMÉRICA LATINA Y EL CARIBE A PARTIR DE EVIDENCIA CUANTITATIVA RECIENTE. Ciudad de Panamá, Panamá: UNICEF

Whitaker, D., Miller, K., \& Clark, L. (2000). Reconceptualizing Adolescent Sexual Behavior: Beyond did they or didn't they? Family Planning Perspectives, 111- 124.

\section{Para citaciones:}

Montero-Mestre, J., Ortíz-Caro, L., \& Castro-Avila, R. (2017). Determinantes socioeconómicos de la frecuencia en las relaciones sexuales en población universitaria. Panorama Económico, 25, 1, pp. 95-114.

\section{AUTORES}

Jorge Montero-Mestre

Integrante del Semillero del Grupo de Investigación Mercado Laboral, Programa de Economía de la Universidad de Cartagena

Leider Ortiz-Caro

Programa de Economía de la Universidad de Cartagena (Colombia)

\section{Robinson Castro-Avila}

Profesor de planta del Programa de Economía e Investigador del Grupo de Investigación Economía Aplicada de la Facultad de Ciencias Económicas de la Universidad de Cartagena (Colombia) 


\section{ANEXOS}

\section{Gráfica 1. Variables cualitativas}

Género Estado Civil del encuestado
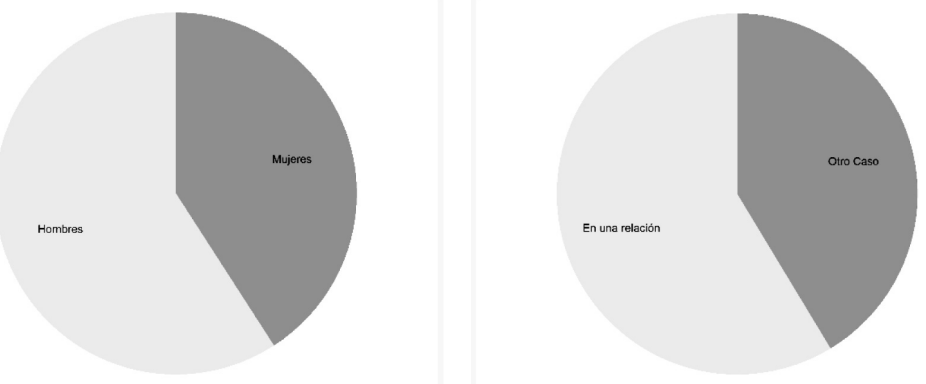

Estado Civil de los Padres Vulnerabilidad de ETS
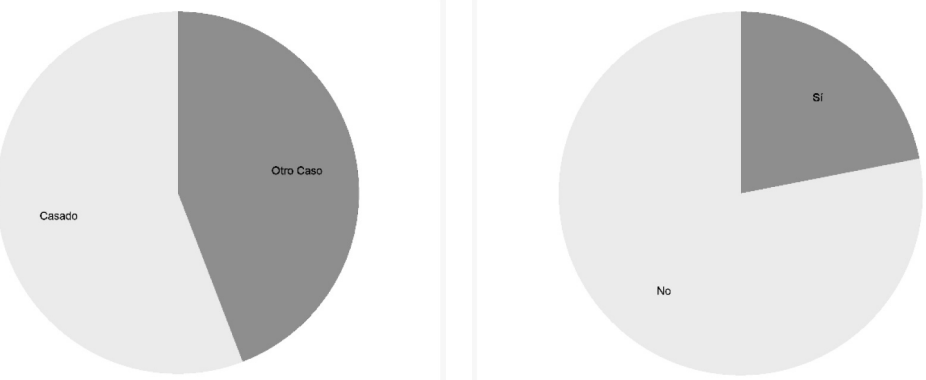

Creencia Educación Sexual
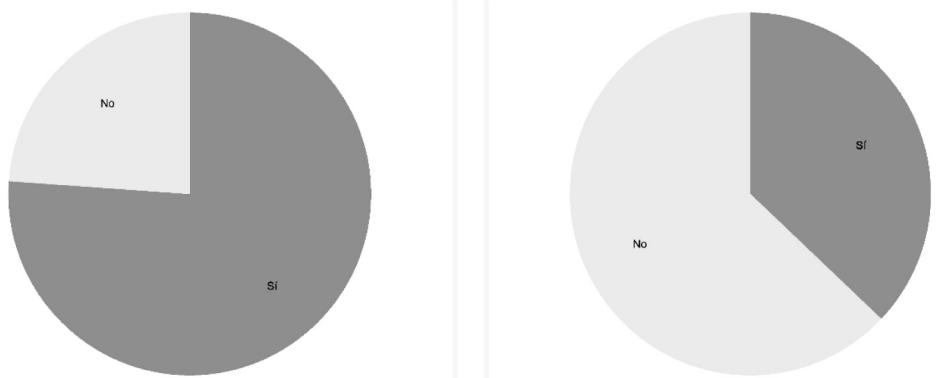

Planificación Consumo de Alcohol

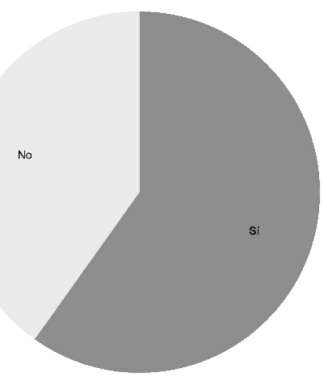

Fuente:Cálculos propios 
Gráfica 2. Relación entre la edad de inicio y la edad del encuestado

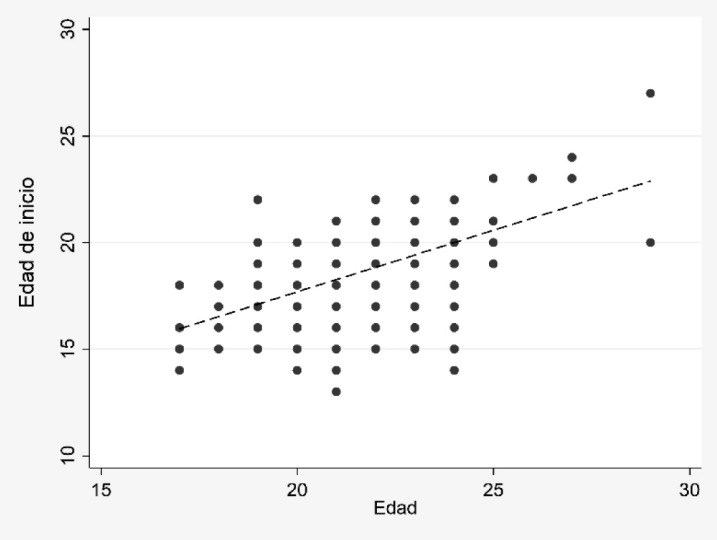

Fuente: Cálculos propios

Gráfica 3. Frecuencia de las relaciones sexuales por sexo y edades
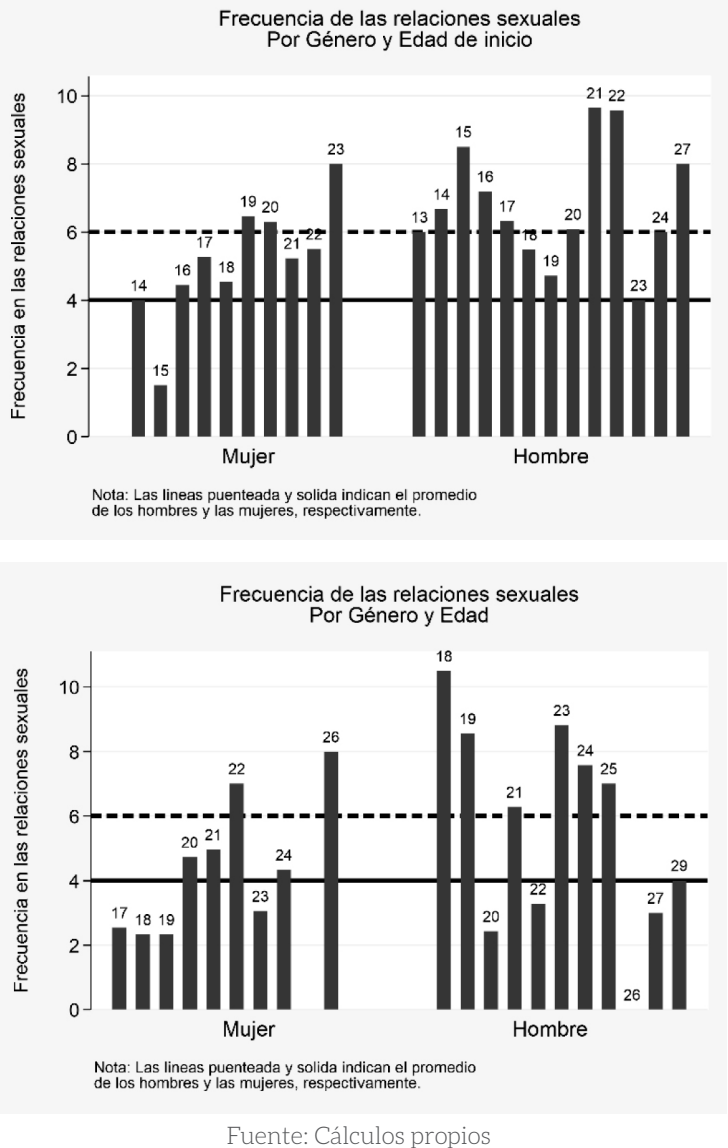
Tabla 2. Estimación de las regresiones

\begin{tabular}{|c|c|c|c|c|}
\hline Variables & MCO & & MCG & \\
\hline \multirow[t]{2}{*}{ Edad } & $-3,225$ & ** & $-3,225$ & ** \\
\hline & $(1,596)$ & & $(1,967)$ & \\
\hline \multirow[t]{2}{*}{ Edad $^{2}$} & 0,083 & ** & 0,083 & * \\
\hline & $(0,036)$ & & $(0,046)$ & \\
\hline \multirow[t]{2}{*}{ Gastos asociados a la Actividad Sexual } & 0,00003 & ** & 0,00002 & *** \\
\hline & $(0,000)$ & & $(7,92 \mathrm{e}-06)$ & \\
\hline \multicolumn{5}{|l|}{ Edad de inicio } \\
\hline \multirow[t]{2}{*}{ Entre 16 y 20} & $-0,931$ & & $-0,931$ & \\
\hline & $(0,882)$ & & $(0,823)$ & \\
\hline \multirow[t]{2}{*}{$>=21$} & $-2,030$ & ${ }^{* * *}$ & $-2,030$ & *** \\
\hline & $(0,748)$ & & $(0,902)$ & \\
\hline \multirow[t]{2}{*}{ Estado Civil } & 0,925 & & 0,925 & \\
\hline & $(0,675)$ & & $(0,645)$ & \\
\hline \multirow{2}{*}{ Estado Civil de los padres } & 1,207 & ** & 1,207 & ** \\
\hline & $(0,575)$ & & $(0,632)$ & \\
\hline \multirow[t]{2}{*}{ Creyente } & 2,092 & *** & 2,092 & *** \\
\hline & $(0,767)$ & & $(0,719)$ & \\
\hline \multirow[t]{2}{*}{ Consumo de Alcohol } & 1,753 & *** & 1,753 & *** \\
\hline & $(0,548)$ & & $(0,668)$ & \\
\hline \multirow[t]{2}{*}{ Planificación } & $-3,229$ & $* * *$ & $-3,229$ & *** \\
\hline & $(0,573)$ & & $(0,689)$ & \\
\hline \multirow[t]{2}{*}{ Constante } & 34,31 & * & 34,31 & ** \\
\hline & $(17,56)$ & & $(20,85)$ & \\
\hline $\mathrm{R}^{2}$ Ajustado & $31,44 \%$ & & & \\
\hline BIC & 1248,480 & & 1248,480 & \\
\hline AIC & 1211,820 & & 1211,820 & \\
\hline $\mathrm{N}$ & 207 & & 207 & \\
\hline
\end{tabular}

Fuente: Cálculos propios..

Nota: En la tabla se encuentran los coeficientes estimados por MCO y MCG. * Significativo al $10 \%$; ** Significativo al $5 \%$; ${ }^{* * *}$ Significativo al $1 \%$. Errores estándar entre parentesis.

Tabla 3. Test Jarque - Bera

\begin{tabular}{|c|c|c|}
\hline Variable & Adj Chi2(2) & Prob > Chi2(2) \\
\hline Resid & 23,8 & 0,0000 \\
\hline
\end{tabular}

Fuente:Cálculos propios

\section{Gráfica 4. Prueba de normalidad del modelo MCG}
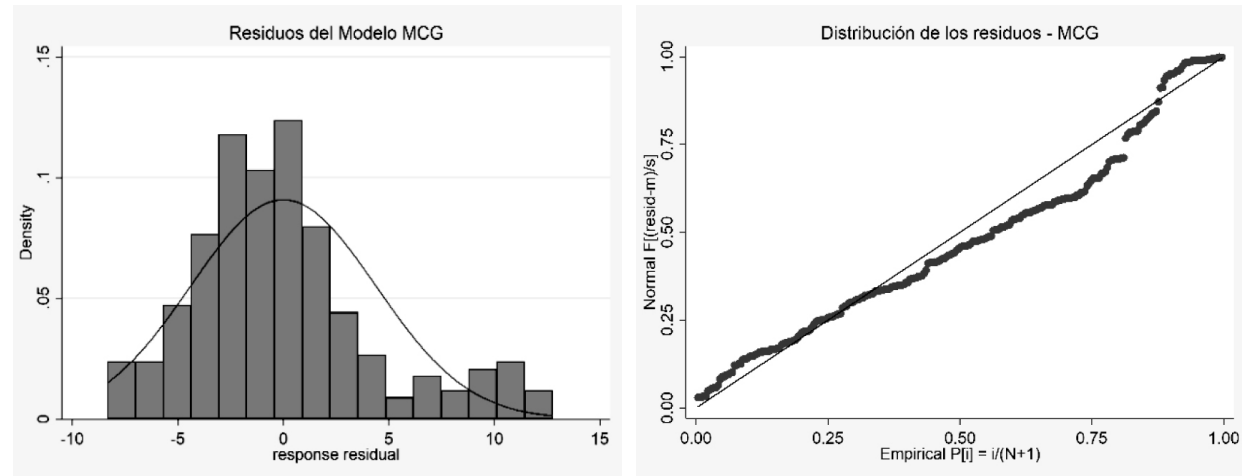

Fuente: Cálculos propios 


\section{Gráfica 5. Efectos Marginales con edades de los encuestados}
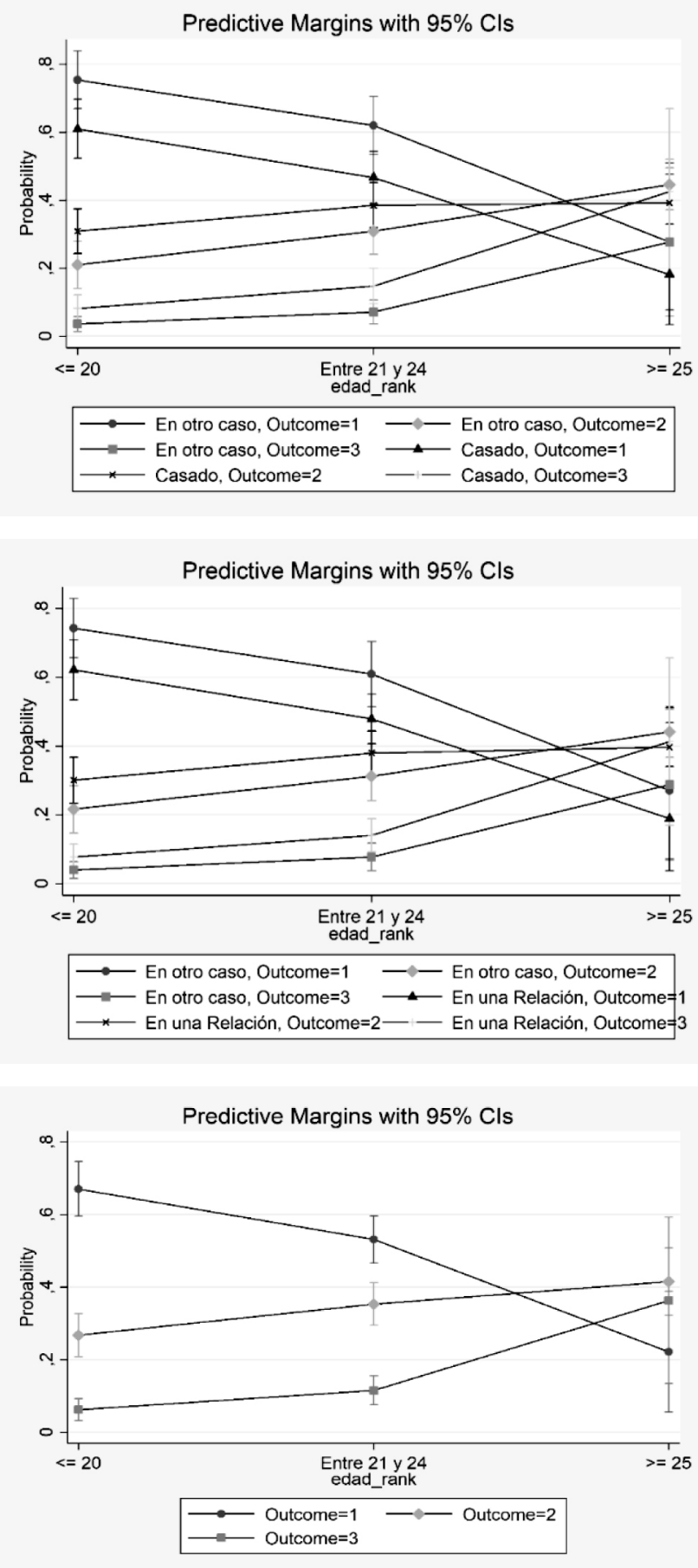

Fuente: Cálculos propios 
Gráfica 6. Efectos Marginales con edades de inicio de relaciones sexuales
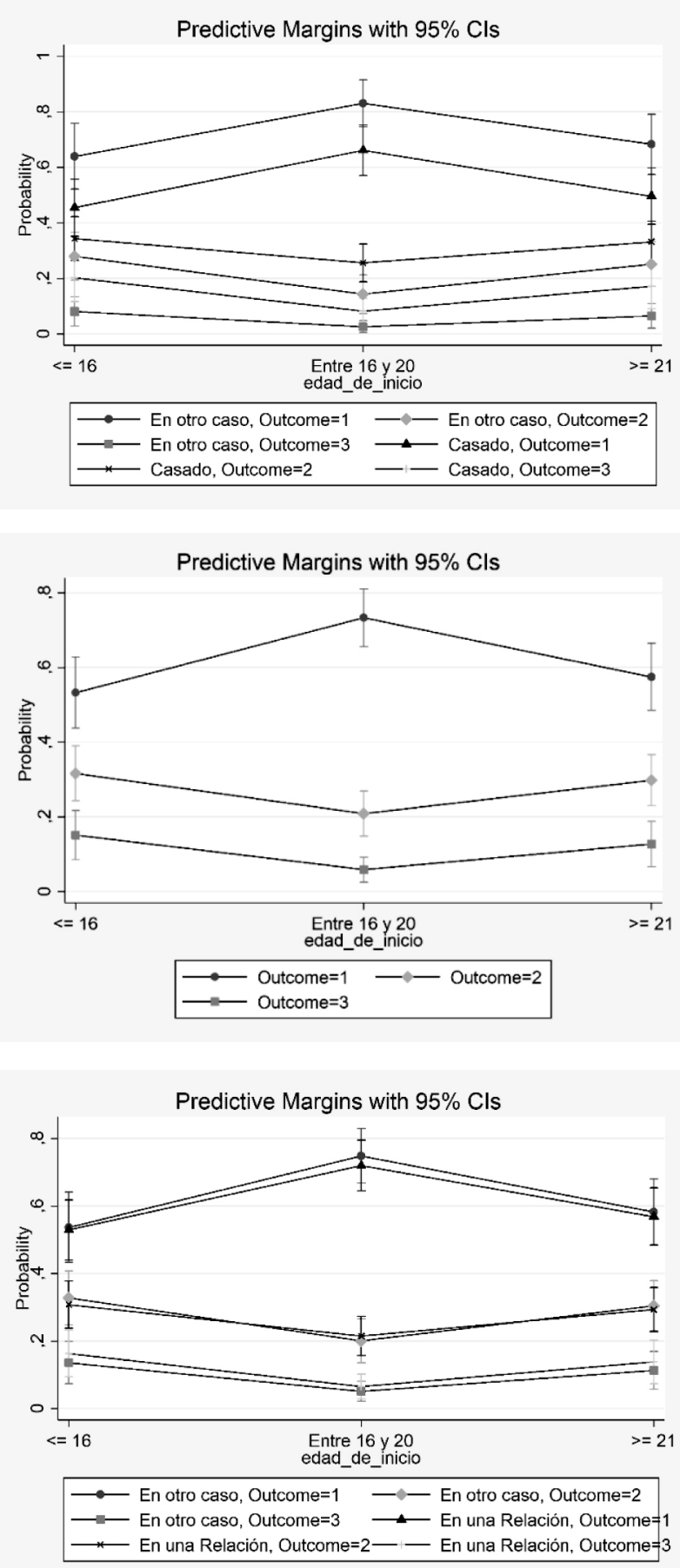

Fuente: Cálculos propios 


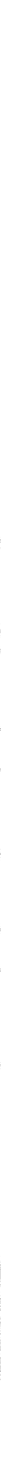

